\title{
Using rsfMRI and ICA in Analysis of Visual Cortex Area in Healthy and Multiple Sclerosis Patients
}

\author{
I. KARPIEL ${ }^{a, *}$ AND Z. DRZAZGA ${ }^{b}$ \\ ${ }^{a}$ Eukasiewicz Research Network - Institute of Medical Technology and Equipment, \\ F.D. Roosevelta 118, 41-800 Zabrze, Poland \\ ${ }^{b}$ Faculty of Science and Technology, University of Silesia in Katowice, \\ A. Chełkowski Institute of Physics, 75 Pułku Piechoty 1, 41-500 Chorzów, Poland \\ Doi: 10.12693/APhysPolA.139.377 *e-mail: ilona.karpiel@itam.lukasiewicz.gov.pl

\begin{abstract}
The aim of this study is to apply the independent component analysis in a GIFT toolbox in a restingstate functional MRI analysis of the visual cortex. The measurements were performed during rest in normal subjects (20) and patients with multiple sclerosis (14) on a $3 \mathrm{~T}$ scanner. A number of independent components influencing the visual network in the MS group in comparison with the healthy group is discussed taking into consideration some spectral parameters such as: dynamic range and low frequency fluctuation and value of kurtosis of time courses and spatial maps. Our analyses have shown that healthy patients are characterized by higher and more numerous activations in the visual areas than MS patients. It should be noted that spectral factors such as kurtosis of spatial maps is markedly higher in the healthy group than in the MS one. The number of activations in the visual area of the brain depends on model order ICA as well as on the kind of the group. The majority of activations in the healthy group were found for ICA100 while in MS high model order $(80,100)$ they were not seen.
\end{abstract}

topics: brain mapping, resting state, ICA, MS

\section{Introduction}

Multiple sclerosis (MS) is an inflammatory and degenerative disease of the central nervous system (CNS) which is an area of ever expanding research and escalating publications. The disease is characterized by multiple lesions principally affecting the white matter (WM), with consequent structural and functional disconnection between various areas of the CNS, resulting in a wide range of symptoms and signs. MS is a progressive disorder that affects the central nervous system. Resting state networks (RSNs) abnormalities have been found in almost all multiple sclerosis phenotypes [1-12]. The disease is presented in many publications [13-20] including those devoted to MRI. Unfortunately, conventional imaging poorly characterizes the degree of injury in demyelinated lesions and does not identify all the pathology in MS [21].

Imaging new modalities have been developed and applied to MS, i.e., MRI, magnetization transfer imaging, spectroscopy, functional MRI, diffusion tensor imaging and resting-state functional MRI (rsfMRI). Functional magnetic resonance imaging (fMRI) during the performance of various tasks has provided a large amount of clinical and neuroimaging data showing functional alterations in MS patients. Recently, there has been more interest in the
rsfMRI method allowing to explore the functional connectivity (FC) of the brain. Brain functional connectivity [22-24] is defined as the coherence in the activity between cerebral areas under a task or in the resting-state (RS), therefore the main purpose of rsfMRI research is to understand alterations in the intrinsic functional architecture of the brain and their role in disease progression and clinical impairment [25-28].

Many previous rsfMRI studies have identified altered functional connectivity expressions and networks from different clinical populations [29-31]. Publications about rsfMRI are rarely presented, especially for the visual area, that is why we decided to show only this area. A generally discussed topic related to this area can be found (excluding multiple sclerosis) in, e.g., [32, 33], where the analysis was used and Group ICA decomposition in 49 and seven relevant independent components of interest, classified into seven functional networks, was presented. For 820 healthy adults, Zhang et al. presented the statistical of FC in occipital networks [34].

Unlike fMRI during task execution, rsfMRI is not influenced by task performance, which may differ from that of healthy subjects, especially in patients with clinical disability. RS-fMRI is a promising tool to study the relationship between spontaneous brain activity and behavioral performance. 
In an earlier study, rsfMRI has been shown to be a promising tool for exploring brain functions relationship between spontaneous brain activity and behavioral performance and assessing their alteration in neurodegenerative conditions [35].

The two main approaches to the analysis of RSNs are based on correlation with the time course in a predefined seed voxel [25, 36-38] and independent component analysis (ICA) [26, 39-42]. ICA is the most common network-based approach to isolate functional networks which is a two-dimensional (time $\times$ voxels) data matrix into a set of time courses and associated spatial maps. Independent components (ICs) are thought to identify synchronous blood oxygen level-dependent (BOLD) signals associated with distinct neural functionalities. ICA mathematically decomposes different independent components reflecting separate intrinsic neural networks, which can be applied to assess the spatiotemporal neural networks and the impact of a particular disease on these networks.

Unfortunately, there are discrepancies in the presented analyses and the selection of components for different disease entities. We focused on the selected visual area because this area is strongly correlated with the motor area which has been subject of our earlier research [43]. The cortical activity is very important for the quality of life both of patients with MS and of healthy people.

We present analyses of the visual cortex and cerebellum for the MS group compared with the healthy subjects using the ICA method with the popular GIFT software. We focus on the presentation of "Infomax" analysis for a selected number of components for both groups.

\section{Image processing and data analysis}

\subsection{Data}

We used data from 14 patients with MS and 20 normal subjects. BOLD fMRI was acquired with a Siemens Magnetom Skyra 3-Tesla scanner using a whole-brain, gradient-echo echo planar sequence with a 32-channel head coil. The measurements of MS patients were acquired in Helimed Diagnostic Imaging in Katowice and the healthy group - in a hospital in Tübingen, Germany. A written informed consent was obtained from each subject. The study was approved by the institutional ethics committee of the University of Silesia and the hospital in Tübingen.

The participants lay supine with their heads snugly fixed by straps and foam pads to minimize head movement. Images were scanned parallel to the anterior commissure-posterior commissure line. The functional images were obtained using an EPI sequence with the following parameters: $\mathrm{TR} / \mathrm{TE}=2000 / 35 \mathrm{~ms}$ for the healthy group and TR 3140 for MS patients, voxel resolution $=3 \times 3 \times 3 \mathrm{~mm}^{3}$, number of time-points $=100$ for MS group and 300 for healthy subjects, 30 axial slices, thickness $=3 \mathrm{~mm}$, flip angle $=90 \mathrm{deg}$, FoV read $190 \mathrm{~mm}$. In addition, a T1-weighted sagittal three-dimensional magnetization-prepared rapid gradient echo (MPRAGE) sequence was acquired, covering the entire brain: 192 slice, $\mathrm{TR}=2300 \mathrm{~ms}, \mathrm{TE}=2.11 \mathrm{~ms}$, slice thickness equals $0.94 \mathrm{~mm}$, flip angle $=8 \mathrm{deg}$, inversion time $=900 \mathrm{~ms}$, FoV read $=240 \mathrm{~mm}$, and in-plane resolution $0.9 \times 0.9 \times 0.9 \mathrm{~mm}^{3}$.

\subsection{Pre-processing}

The functional and anatomical image manual coregistration was performed using a SPM8 (statistical parametric mapping) toolbox under MATLAB $\mathrm{R} 2014 \mathrm{~b}$ software. The first three images were discarded. After removing the files, functional images were manually coregistered and point $(0,0,0)$ in point anterior commissure (AC) for each subject was set. Subsequently, data were processed by using GIFT.

\subsection{Post-processing}

Post-processing was performed using the spatial independent component analysis using the GIFT software [44]. In our work, we used the scheme of the rsfMRI data analysis according to Fig. 1. For each subject, pre-processed data were decomposed into a chosen number of $20,40,60,80,100$ ICs by using the Infomax algorithm and analysis because the number of ICs and other algorithms in many publications is chosen very freely. There is a lack of knowledge about the effect of increasing model order on ICs' characteristics of RSNs. For each of those components, characteristic time courses and spatial maps were generated. For each of the patients, we calculated the fractional amplitude of low-frequency fluctuation (fALFF) to characterize the regional spontaneous activity in a voxel-wise way using the GIFT toolbox.



Fig. 1. Scheme of the rsfMRI data analysis. 
Group analysis with the highest value of fALFF and dynamic range for the healthy group.

TABLE I

\begin{tabular}{l|c|c|c|c|c}
\hline \hline & ICA20 & ICA40 & ICA60 & ICA80 & ICA100 \\
\hline Number of components & 18 & 19 & 35 & 1 & 24 \\
Coordinate & $0.05,-88.5,0.5$ & $-6.5,-53.5,0.5$ & $-9.5,-62.5,2.5$ & $-0.5,-43.5,-10.5$ & $8.5,-82.5,27$ \\
Dynamic range & 0.03074 & 0.030313 & 0.030635 & 0.023736 & 0.029379 \\
fALFF & 4.175 & 3.5451 & 3.8167 & 1.8322 & 3.7162 \\
\hline Number of components & 19 & 28 & 48 & 54 & 44 \\
Coordinate & $-2.5,-75.5,27.5$ & $0.5,-89.5,7.5$ & $0.5,-85.5,-2.5$ & $-9.5,-62.5,2.5$ & $21.5,-92.5,14.5$ \\
Dynamic range & 0.031568 & 0.032045 & 0.030542 & 0.026954 & 0.029281 \\
fALFF & 3.5034 & 4.8957 & 4.5956 & 2.9545 & 4.3222 \\
\hline Number of components & & 39 & 59 & 59 & 61 \\
Coordinate & & $-3.5,-79.5,27.5$ & $-3.5,-79.5,27.5$ & $8.5,-81.5,24.5$ & $26.5,-69.5,-35.5$ \\
Dynamic range & 0.030336 & 0.029008 & 0.027829 & 0.025704 \\
fALFF & 3.8778 & 3.4926 & 3.6199 & 2.166 \\
\hline Number of components & & & & & 65 \\
Coordinate & & & & $-9.5,-52.5,0.5$ \\
Dynamic range & & & & 0.024486 \\
fALFF & & & & 2.0894
\end{tabular}

TABLE II

Group analysis with the highest value of fALFF and dynamic range for the MS group (only the occipital lobe).

\begin{tabular}{l|c|c|c}
\hline \hline & ICA20 & ICA40 & ICA60 \\
\hline Number of components & 15 & 37 & 45 \\
Dynamic range & 0.05682 & 0.065294 & 0.062494 \\
fALFF & 1.0952 & 1.5956 & 1.5393
\end{tabular}

From the remaining components, only voxels exceeding an arbitrarily set $z$-score cutoff of 1 were visualized as "activated" and finally we focused on the occipital lobe and visualization results in the GIFT toolbox. Finally, we presented and compared kurtosis (a dimensionless statistical measure to describe the non-Gaussian nature of an arbitrary probability distribution that quantifies over-concentration of observed values in the far tails of some reference distribution) for each person (see Tables I and II).

\section{Results - ICA}

Based on the obtained analysis, components with the highest values of fALFF were selected $(>1)$ for MS and for healthy subjects (Tables I, II). Next, we focused on the analysis of these components which contain areas of our interest, the areas of the occipital and cerebellum. The cerebellum was taken into consideration because it coordinates voluntary movements such as posture, balance, coordination, and speech which is very important.

Spatial maps of the analysis are shown in Fig. 2 and coordinates of their peak activations are provided in Tables I and II for the healthy and MS groups.

Table I shows the selected components for the visual and cerebellum area for the healthy group.
The majority of components were obtained using ICA100, while the highest values in this area were obtained for ICA40 (number of components: 28). Regardless of the number of components selected, the cerebellum and occipital areas of activations were located. A much smaller number of components with lower values were obtained for the MS group.

It follows from Table II that the marked occipital activity in the MS group was received only for three components (ICA 20, Ics15, ICA40, Ics37 and ICA60, Ics45). The highest value for the conducted analyses is 1.5 for fALFF while the fALFF values for the healthy group are nearly three time larger than for the MS patients.

HEALTHY GROUP

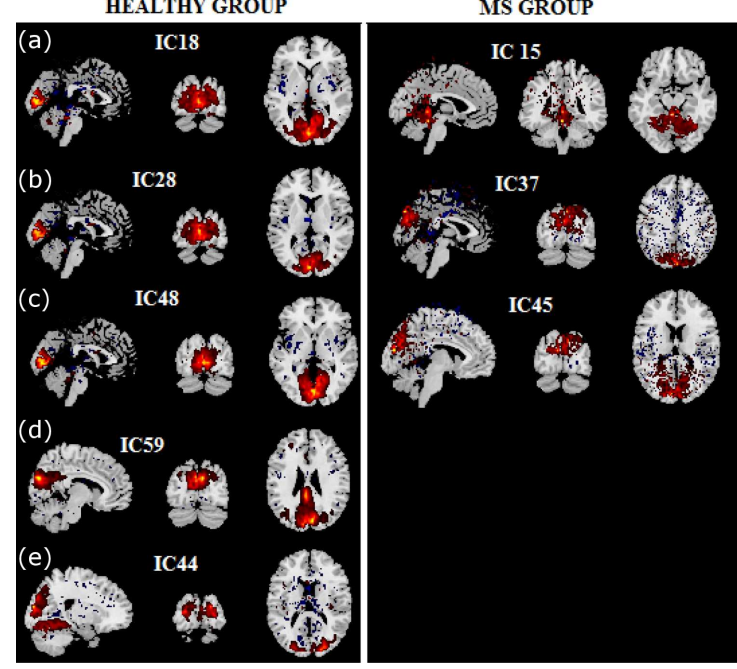

Fig. 2. Compared results of visual networks for the healthy and MS group. Infomax algorithm with (a) ICA20, (b) ICA40, (c) ICA60, (d) ICA80, (e) ICA100. 

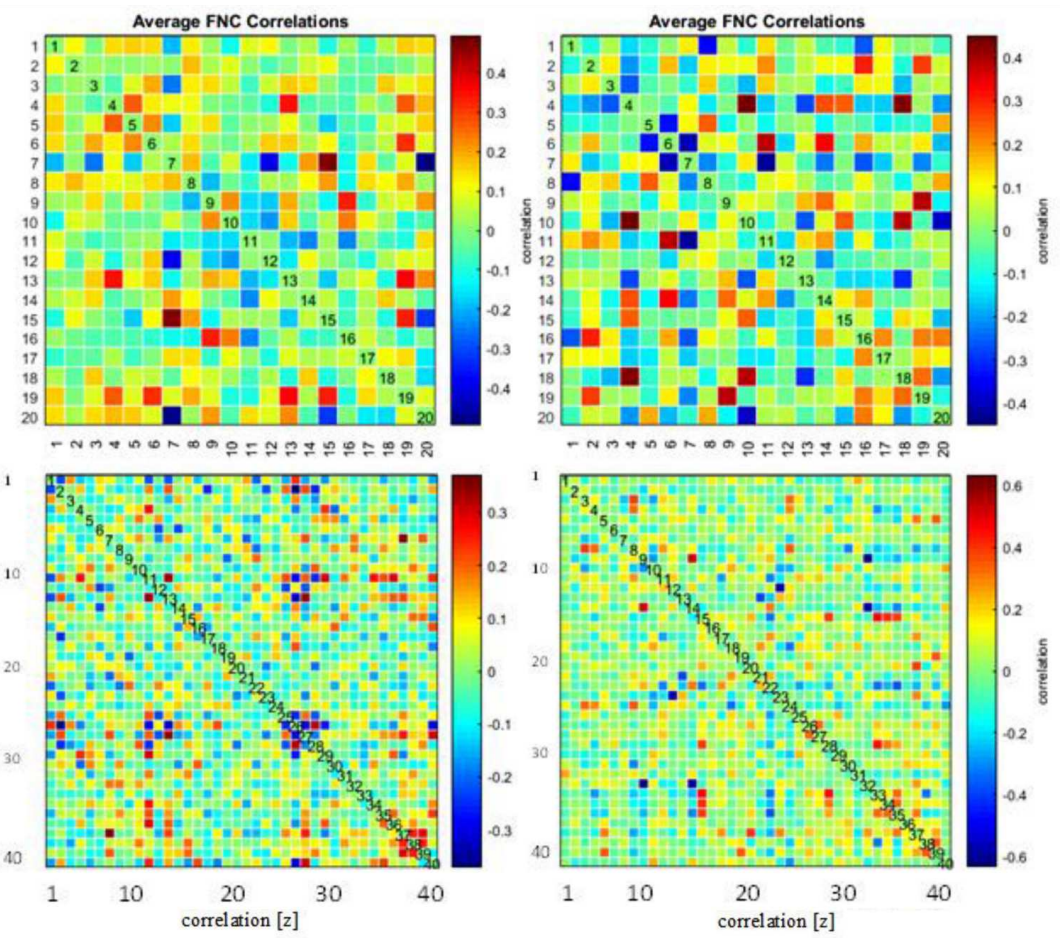

Fig. 3. Example of FNC full correlations of resting state networks for 20 and 40 algorithm Infomax (ICA20, ICA 40). Functional network connectivity correlations are computed for each data-set and averaged across sessions for 14 subjects with MS (a, c) and 20 healthy (b, d) subjects for $z$-value presented.

Figure 2 presents the comparison of localization of visual areas with the highest values of fALFF between the MS and healthy groups for using the ICA method regardless of the declared number of components. For the healthy group, strong activations were found for all the studied number of components: ICA 20 (low model order), 40, 60, 80 and 100 (high model orders). Components IC18, IC28, IC48, IC59 allowed to locate the activation in the same place in the brain. In the case of IC44 for ICA 100 , activations were obtained in the visual area and cerebellum (coordinates are placed in Tables I and II). For the MS group, only the three component values - component IC 15 for ICA20, IC37 for ICA40 and IC45 for ICA60 - were found. Unfortunately, the application for more components, i.e., of ICA 80 and ICA100, did not allow locating the visual area.

It follows from Tables I and II that values for kurtosis of time courses are relatively stable for both groups (healthy and MS), unlike spatial maps.

Figure 3 shows FNC full correlations of resting state networks for 20 and 40 algorithm Info$\max ($ ICA20, ICA 40). For instance, based on a 20-component analysis, strong positive correlations were observed between the networks (IC No. 7 and IC No. 15) for the MS group and (IC No. 4 and IC No. 18) for the healthy group while for ICA 40, positive correlations were observed between (IC 39 and IC 19), (IC 28 and IC15) - the healthy group, (IC 37 and IC 27), (IC 13 and ICA 27 ) the MS group. It is interesting that for ICA40 and
ICA60, higher values of FC for the healthy group (up to 0.6) than for ICA20 (up to 0.4) were obtained, unlike the MS subject.

\section{Discussion}

It was presented in, e.g., [45, 46], that studies, with fMRI, have consistently demonstrated functional cortical changes in the major multiple sclerosis (MS) clinical phenotypes. fMRI during the performance of various tasks has provided a large amount of data showing functional alterations in MS patients [47]. Modifications were characterized by an altered recruitment of regions normally devoted to the performance of a given task, the recruitment of additional areas. Recently, researchers have demonstrated the usefulness of rsfMRI for MS diagnosis taking into consideration a different region of the brain [10]. Unfortunately, very few publications concern the visual area in the MS group.

On the other hand, differences in the functional connectivity of the primary visual area between individuals with amblyopia and normal-sighted subjects using the resting-state functional magnetic resonance imaging were reported [48]. Recently, a new work appeared presenting the rs functional connectivities within the primary visual cortex and between the primary visual cortex and other cortical areas [49] for the subjects with strabismic and a normal control group. Analyses on a group of patients with the eyes open and with the eyes closed [50] 
showed that no significant between-condition differences in connectivity strength were found in the default mode, attention, visual, and motor networks. Dong et al. focused on evaluating the split-half reproducibility of the rsfMRI measures in detecting changes of the resting state activity between eyes open and eyes closed [51]. Our work focused only on analyzing patients with the eyes closed for both studied groups (the MS group and the healthy group).

Our analyses have shown that healthy patients are characterized by higher activations in the visual areas than the MS ones. It should be noted that single and small activity (fALFF: 1-1.6) in the visual areas in the MS group was observed (see Table II). In addition, it is difficult to locate the visual area in the use of ICA 80 or ICA 100 - in these cases the analysis did not bring results. For healthy subjects, components with higher fALFF were stronger and (fALFF: 3-4.8) numerous (see Table I). In [52], it was mentioned that the highest values for the visual area were located for six components using a 75-component GICA.

It should be noted that spectral factors, such as kurtosis of spatial maps, are markedly higher in the healthy than in the MS group. In our previous work, differences in kurtosis of spatial maps and kurtosis were found for the sensimotor cortex [43].

Our results provide functional organization in response to MS which can be analyzed using algorithm Infomax. ICA20 seems to be useful as a preliminary test, while more numerous-component ICA (ICA40, 60) shows more components with higher values of fALFF refining research. In addition, spectral factors such as fALFF, kurtosis of spatial maps parametric determinants of rsfMRI were considered for the studied groups.

\section{Conclusion}

ICA as a relatively fast and widely available method can be successfully used in the diagnosis of MS. The visual area in the MS group showed a smaller number of activation areas than in the healthy group and characterized lower parameters fALFF and higher values of kurtosis of spatial maps in comparison with the healthy subjects.

\section{Acknowledgments}

The authors (Z.D. and I.K.) would like to thank Dr. Aldona Giec-Lorenz from Helimed Diagnostic Imaging in Katowice for enabling the use of rsfMRI data.

\section{References}

[1] S.D. Roosendaal, M.M. Schoonheim, H.E. Hulst, E.J. Sanz-Arigita, S.M. Smith, J.J.G. Geurts, F. Barkhof, Brain 133, 1612 (2010).
[2] Y. Liu, Y. Duan, P. Liang et al., Acta Radiol. 53, 1073 (2012).

[3] D.J. Hawellek, J.F. Hipp, C.M. Lewis, M. Corbetta, A.K. Engel, Proc. Natl. Acad. Sci. USA 108, 19066 (2011).

[4] M.A. Rocca, P. Valsasina, V. Martinelli, P. Misci, A. Falini, G. Comi, M. Filippi, Neurology 79, 1449 (2012).

[5] A. Faivre, A. Rico, W. Zaaraoui et al., Mult. Scler. 18, 1251 (2012).

[6] F. Tona, N. Petsas, E. Sbardella, L. Prosperini, M. Carmellini, C. Pozzilli, P. Pantano, Radiology 271, 814 (2014).

[7] A.-M. Dogonowski, H.R. Siebner, P. Soelberg Sorensen, O.B. Paulson, T.B. Dyrby, M. Blinkenberg, K.H. Madsen, Acta Neurol. Scand. 128, 328 (2013).

[8] Y. Liu, P. Liang, Y. Duan et al., J. Neurol. Sci. 304, 127 (2011).

[9] M. Filippi, M.A. Rocca, Expert Rev. Neurotherap. 13(12), 27 (2013).

[10] S. Bonavita, A. Gallo, R. Sacco et al., Mult. Scler. 17, 411 (2011).

[11] B. Basile, M. Castelli, F. Monteleone, U. Nocentini, C. Caltagirone, D. Centonze, M. Cercignani, M. Bozzali, Multiple Sclerosis J. 20, 1050 (2014).

[12] M.A. Rocca, P. Valsasina, M. Absinta et al., Neurology 74, 1252 (2010).

[13] F. Barkhof, M. Rocca, G. Francis et al., Ann. Neurol. 53, 718 (2003).

[14] W.I. McDonald, A. Compston, G. Edan et al., Ann. Neurol. 50, 121 (2001).

[15] C.H. Polman, S.C. Reingold, G. Edan et al., Ann. Neurol. 58, 840 (2005).

[16] C.M. Dalton, P.A. Brex, K.A. Miszkiel, S.J. Hickman, D.G. MacManus, G.T. Plant, A.J. Thompson, D.H. Miller, Ann. Neurol. 52, 47 (2002).

[17] CHAMPS Study Group, Neurology 59, 998 (2002).

[18] M. Tintoré, A. Rovira, J. Río et al., Neurology 60, 27 (2003).

[19] A. Charil, T.A. Yousry, M. Rovaris et al., Lancet Neurol. 10, 841 (2006).

[20] D.H. Miller, B.G. Weinshenker, M. Filippi et al.. Mult. Scler. 14, 1157 (2008).

[21] J.R. Fox, E. Beall, P. Bhattacharyya, T.J. Chen, K. Sakaie, Neurol. Clin. 29, 357 (2011).

[22] B. Biswal, F.Z. Yetkin, V. M. Haughton, J.S. Hyde, Magn. Reson. Med. 34, 1910340409 (1995).

[23] B.B. Biswal, Neuroimage 62, $938 \mathrm{e} 944$ (2012). 
[24] A.Z. Snyder, M.E. Raichle, Neuroimage 62, 902e910 (2012).

[25] M.D. Fox, A.Z. Snyder, J.L. Vincent, M. Corbetta, D.C. Van Essen, M.E. Raichle, Proc. Natl. Acad. Sci. USA 102, 9673 (2005).

[26] J. Damoiseaux, S. Rombouts, R. Barkhof, P. Scherltens, C. Stam, S. Smith, C. Beckmann, Proc. Natl. Acad. Sci. USA 103, 848 (2013).

[27] R. Li, K. Chen, A.S. Fleischer, E.M. Reiman, L. Yao, X. Wu, Neuroimage 56, 1035 (2011).

[28] J.M. Lowe, Neuroimage 62, 1146 (2012).

[29] R.A.T. Dosenbach et al., Proc. Natl. Acad. Sci. USA 104, 11073 (2007).

[30] M. Greicius, Curr. Opin. Neurol. 21, 424 (2008).

[31] A. Fornito, A. Zalesky, C. Pantelis, E.T. Bullmore, Neuroimage 62, 2296 (2012).

[32] B. Rashid, E. Damaraju, G.D. Pearlson, V.D. Calhoun, Front. Hum. Neurosci. 8, 897 (2014).

[33] B. Rashid, J. Chen, I. Rashid et al., Neuroimage 184, 843 (2018).

[34] C. Zhang, S.A. Baum, V.R. Adduru, B.B. Biswal, A.M. Michael, Neuroimage 183, 907 (2018).

[35] F. Barkhof, S. Haller, S.A. Rombouts, Radiology 272, 29 (2014).

[36] B.B. Biswal, J.V. Kylen, J.S. Hyde, NMR Biomed. 10, 165 (1997).

[37] D. Cordes, V.M. Haughton, K. Arfanakis, G.J. Wendt, P.A. Turski, C.H. Moritz, M.A. Quigley, M.E. Meyerand, AJNR Am. J. Neuroradiol. 21, 1636 (2000).

[38] D. Cordes, V.M. Haughton, K. Arfanakis, J.D. Carew, P.A. Turski, C.H. Moritz, M.A. Quigley, M.E. Meyerand, AJNR Am. J. Neuroradiol. 22, 1326 (2001).
[39] V.D. Calhoun, T. Adali, IEEE Eng. Med. Biol. Magn. 25, 79 (2006).

[40] M. Greicius, B. Krasnow, A. Reiss, V. Menon, Proc. Natl. Acad. Sci. USA 100, 253 (2003).

[41] B. Harrison, J. Pujol, H. Ortiz, A. Fornito, C. Pantelis, M. Yücel, PLoS ONE 3, e1794 (2008).

[42] R. Szupiluk, T. Ząbkowski, K. Gajowniczek, Acta Phys. Pol. A 127, A-139 (2015).

[43] I. Karpiel, Z. Drzazga, in: Advances in Intelligent Systems and Computing, Eds. M. Gzik, Z. Paszenda, E. Piętka, E. Tkacz, K. Milewski, Springer Int. Publ., 2020, p. 1 (in press).

[44] Group ICA Of fMRI Toolbox (GIFT).

[45] M. Filippi, F. Agosta, E.G. Spinelli, M.A. Rocca, J. Neurol. 260, 1709 (2013).

[46] M. Filippi, M.A. Rocca, Expert Rev. Neurotherap. 13, 27 (2013).

[47] P. Pantano, C. Mainero, F. Caramia, J. Neuroimag. 16, 104 (2006).

[48] Kun Ding, Yong Liu, Xiaohe Yan, Xiaoming Lin, Tianzi Jiang, Neural Plastic. 2013, 612086 (2013).

[49] X. Yan, Y. Wang, L. Xu, Y. Liu, S. Song, K. Ding, Y. Zhou, T. Jiang, X. Lin, Curr Eye Res. 44, 316 (2019).

[50] R. Patriat, E.K. Molloy, T.B. Meier, G.R. Kirk, V.A. Nair, M.E. Meyerand, V. Prabhakaran, R.M. Birn, Neuroimage 78, 463 (2013).

[51] Dong-Qiang Liu, Zhangye Dong, Xinian Zuo, Jue Wang, Yufeng Zang, Neuroinformatics 11, 469 (2013).

[52] E.A. Allen, E.B. Erhardt, Eswar Damaraju et al., Front. Syst. Neurosci. 5, 2 (2011). 